\title{
Evaluation de la commercialisation et de la consommation du poisson fumé au Congo: cas de Brazzaville
}

\author{
MANANGA Vital ${ }^{(1,2,3)}$, ITOUA OKOUANGO Yvon Simplice ${ }^{(1,2)}$, ZOLA TCHINA \\ Patience Amelia ${ }^{(2)}$, ELENGA Michel ${ }^{(1,2)}$ \\ (1)Laboratoire de Nutrition et d'Alimentation Humaine (LaNAH), Faculté des Sciences et Techniques (FST), UMNG, BP : \\ 69, Brazzaville, Congo. \\ (2)Equipe Pluridisciplinaire de Recherche en Alimentation et en nutrition (EPRAN) Faculté des Sciences et Techniques (FST), \\ UMNG. \\ (3) Auteur correspondant: MANANGA Vital, docteur en alimentation et nutrition, Département de Biologie et Physiologie \\ Animales, Faculté des Sciences et Techniques (Université Marien Ngouabi), BP : 69, Brazzaville, Congo. \\ E-mail : manangavital@yahoo.fr, tel : 00(242) 066743151/00(242) 057038912
}

Mots clés : enquête, consommation ; vente, poisson fumé ; Brazzaville

Keywords: survey, consumption; sale, smoked fish; Brazzaville

Publication date 31/07/2019, http://www.m.elewa.org/JAPS

\section{RÉSUMÉ}

Le poisson fumé constitue une source importante de protéines, accessibles aux ménages à faible revenus. L'objectif général de cette étude est d'évaluer la consommation des poissons fumés et de connaître les différents types de poisson fumés vendus et consommés à Brazzaville. Deux enquêtes ont été réalisées. Une première enquête exhaustive a porté sur l'inventaire des différents types des poissons fumés vendus auprès de 208 vendeurs de poissons fumés recensés dans trois marchés de Brazzaville (Mampassi, Moungali et Total). Une deuxième enquête transversale a porté sur la consommation du poisson fumé auprès de 120 ménages des deux arrondissements (Bacongo et Ouénzé). S'agissant de l'inventaire, les résultats montrent que 23 types de poissons fumés sont vendus dans les marchés enquêtés. Les poissons les plus vendus sont : Protopterus dolloï (62,50\%), Distichodus spp $(48,55 \%)$ et Clarias spp (45,19\%). Appelé respectivement en langue vernaculaire (lingala): « Nzombo ", « Mboto » et« Ngolo ». Concernant la consommation, les poissons fumés sont largement consommés dans les ménages de Brazzaville $(87,5 \%)$. dont les plus consommés sont: Clarias spp« Ngolo ", Protopterus dollö Nzombo »et Distichodus spp« Mboto » avec une fréquence de consommation qui varie d'une à 2 fois et plus par semaine. L'enquête a révélé que le poisson d'eau douce fumé occupe une place de choix aussi bien, dans le panier de la ménagère et à la consommation alimentaire des habitants de Brazzaville. Il assure par conséquent, une bonne couverture de la sécurité alimentaire auprès de ladite population.

\section{SUMMARY}

Smoked fish is an important source of protein, accessible to low-income households. The general objective of this study is to evaluate the consumption of smoked fish and to know the different types of smoked fish sold and consumed in Brazzaville. Two surveys were conducted. A first exhaustive survey focused on the inventory of different types of smoked fish sold to 208 smoked fish vendors listed in three markets of Brazzaville (Mampassi, Moungali and Total). A second cross-sectional survey focused on the consumption of 
smoked fish among 120 households in the two boroughs (Bacongo and Ouénzé). With regard to the inventory, the results show that 23 types of smoked fish are sold in the surveyed markets. The best-selling fish are: Protopterus dolloï (62.50\%), Distichodus spp $(48.55 \%)$ and Clarias spp (45.19\%). Called respectively in vernacular language (lingala): "Nzombo", "Mboto" and "Ngolo". Regarding consumption, smoked fish are widely consumed in households in Brazzaville $(87.5 \%)$. of which the most consumed are: Clarias spp. "Ngolo", Protopterus dolloï "Nzombo" and Distichodus spp "Mboto" with a consumption frequency that varies from one to two times and more per week. The survey revealed that smoked freshwater fish occupy a prominent place both in the household basket and in the food consumption of the inhabitants of Brazzaville. It ensures, therefore, a good coverage of food security with the said population.

\section{INTRODUCTION}

Le poisson est un aliment naturel dont l'homme s'est sustenté depuis la nuit des temps. Très tôt, il a été reconnu comme favorable à un bon équilibre physiologique (Diènèba, 2005). En Afrique, les populations font souvent recours aux espèces animales dont les poissons pour couvrir leurs besoins et assurer la sécurité alimentaire (World Fish center, 2005). Le poisson fumé constitue une source importante de protéines, accessibles aux ménages à faibles revenus, surtout dans les pays en voie de développement où le prix de la viande demeure hors de portée du consommateur moyen (FAO, 2009). En 2003, la FAO estime que le poisson représente $22 \%$ de la ration protéinique en Afrique subsaharienne. Cependant, dans les pays les plus pauvres, ce taux peut dépasser 50 $\%$, en particulier lorsque les autres sources en protéines animales sont rares ou chères. En Afrique, plus de 200 millions de personnes consomment du poisson régulièrement frais, mais plus souvent fumé ou séché (World Fish center, 2005). Au Benin, les trois quarts de la production halieutique sont consommés frais, le reste est fumé, séché, salé, ou frit avant d'être distribué sur les marchés intérieurs du pays (Gbaguidi et Fiogbé, 1998). Par ailleurs, le poisson contribue aussi indirectement à l'autosuffisance alimentaire nationale, du fait des échanges commerciaux et de l'exportation (World Fish center, 2005). En 2000 la FAO rapporte que les produits de la pêche sont en grande partie commercialisés vers les centres urbains, car ils constituent l'une des rares sources de revenus des populations rurales. En effet, les poissons, par leur valeur nutritive, constituent un complément précieux dans les régimes alimentaires pauvres en protéines, en vitamines et en sels minéraux (FAO, 2006). Cependant, plusieurs études ont signalé que les poissons sont des denrées périssables, avec une vitesse d'altération élevée (Gram, 1987 ; Liston, 1992). Les pêcheurs font surtout recours aux procédés de conservation notamment le fumage pour prolonger la durée de conservation du poisson et assurer sa disponibilité. Semble doute, à Brazzaville, le poisson fumé est retrouvé dans les recettes et dans les marchés, mais le taux de vente et de consommation, ainsi que la fréquence de sa consommation sont moins connus. Il existe une diversité de poissons fumés vendus et consommés à Brazzaville. Les poissons fumés sont souvent consommés par les ménages avec une importance variable. Notre étude s'inscrit dans le cadre d'une meilleure visibilité de la place du poisson fumé dans les habitudes alimentaires des habitants de Brazzaville. Il s'agit d'Inventorier les poissons fumés vendus à Brazzaville, connaittre les poissons fumés consommés à Brazzaville et évaluer la consommation dudit poisson. 
3

3.1 Cadre d'étude: Notre étude a eu pour cadre les marchés de Brazzaville notamment : Total, Mampassi, et Moungali, les ménages de Brazzaville notamment ceux des arrondissements de Bacongo et Ouénzé et le laboratoire de Nutrition et Alimentation Humaines de la Faculté des Sciences et Techniques.

3.2 Description du lieu d'étude : Brazzaville, première ville, est la capitale politique et administrative de la République du Congo. Elle se situe dans le sud du Congo, sur la droite rives du fleuve Congo, sur la rive nord du Pool Malebo en face de Kinshasa, capitale de la République Démocratique du Congo. Sa superficie est de 269,3 $\mathrm{km}^{2}$. La population actuelle de Brazzaville atteint environ 1.696.392 d'habitants. Brazzaville compte 9 arrondissements parmi lesquels on citera ceux qui feront l'objet de notre étude.

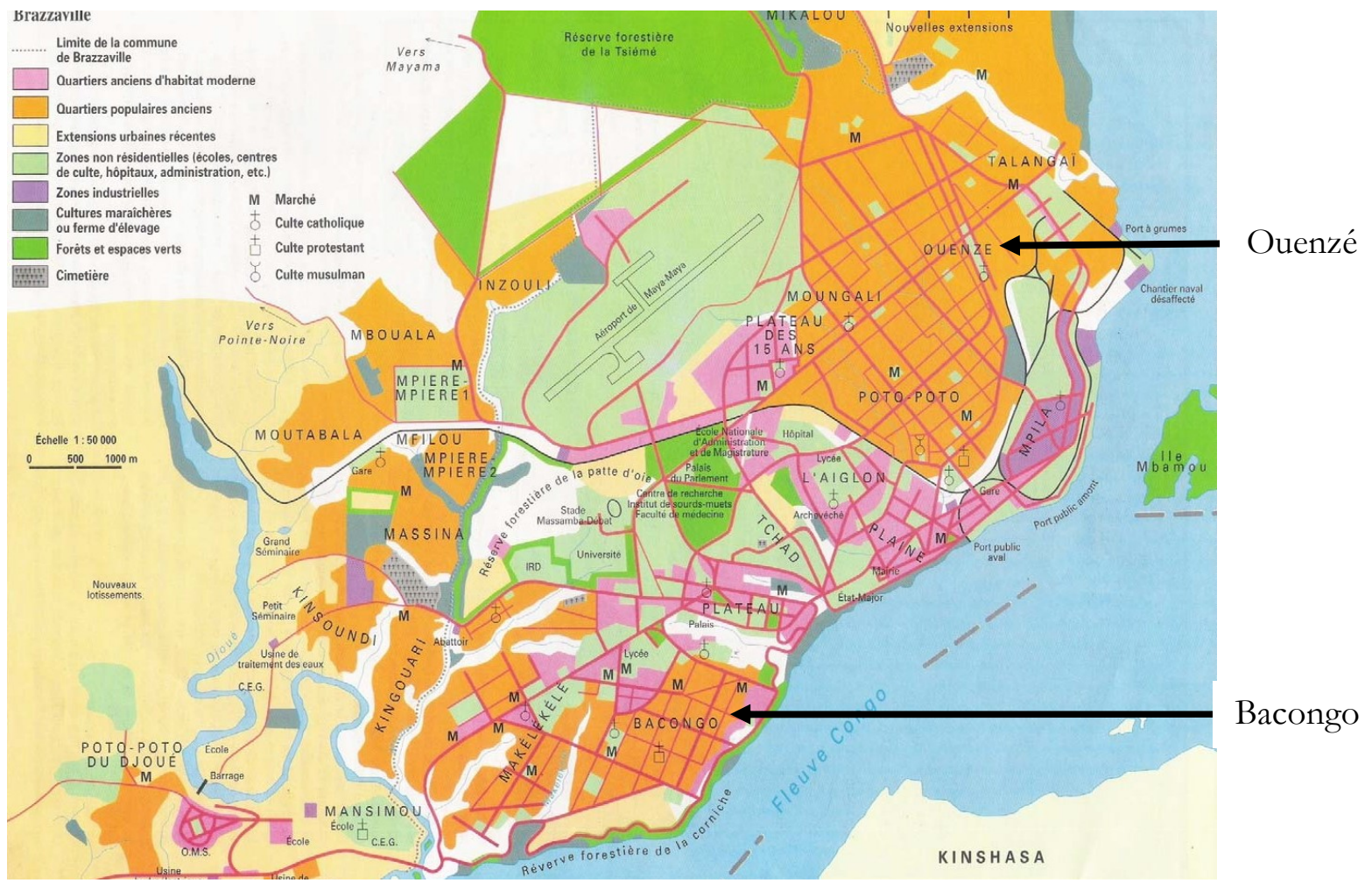

Figure 1 : Carte de la ville de Brazzaville (Fray R., 1980)

3.3 Matériel didactique : Les informations ont été collectées à partir :

* D'une fiche pour l'inventaire des poissons fumés vendus dans les marchés.

* D'une fiche de consommation des poissons fumés dans les ménages et /ou famille.

Les informations recueillies ont porté sur les renseignements généraux ; la consommation du poisson fumé, le mode de consommation ou de préparation, la fréquence de consommation du poisson fumé et l'appréciation du poisson fumé sur le plan organoleptique.

\subsection{METHODES}

3.4.1 Travail sur le terrain: Le travail sur le terrain est caractérisé par deux enquêtes: une enquête d'inventaire des poissons fumés dans les marchés et une enquête de consommation des poissons fumés, dans les ménages.

3.4.2 Enquête sur la commercialisation du poisson fumé dans les marchés: Nous avons réalisé une enquête d'inventaire dans trois marchés de Brazzaville : Total, Mampassi et Moungali. Elle a consisté à inventorier les différents types de poissons fumés vendus et a permis de connaitre les 
poissons les plus vendus dans ces marchés. Les informations ont été recueillies à partir d'une fiche d'inventaire (annexe 1), avec comme variable étudiée : les types de poissons fumés vendus. Cette enquête a été réalisée sur tous les vendeurs de poissons fumés (étalages des rayons des poissons fumés institués par la mairie). Il s'agit d'une enquête exhaustive.

3.4.3 Type d'étude : Il s'agit d'un recensement des types de poissons fumés vendus dans les trois marchés sélectionnés (Total, Mampassi et Moungali).

3.4.4 Population cible : La population cible est constituée des vendeurs de poissons fumés dans les marchés Total, Mampassi et Moungali.

\subsubsection{Critères d'inclusion}

- $\quad$ Accepter de participer à l'enquête ;

- Vendre les poissons fumés dans les

marchés sélectionnés (Total, Mampassi et Moungali) ;

- Vendre les poissons fumés sur les étalages des rayons des poissons fumés institués par la mairie.

3.5 Déroulement de l'enquête : L'enquête dans les marchés s'est déroulée pendant trois jours (1jours /marché). Dans chaque marché, une zone d'étude a été délimitée (zone de vente des poissons fumés prévus par la mairie). Un inventaire des différents types de poissons fumés retrouvés sur chaque étalage a été réalisé. Ce qui a permis de trouver 208 étalages correspondant à 208 vendeurs de poissons fumés dans l'ensemble des marchés enquêtés. Les vendeurs de poissons fumés ont été interrogés sur les noms des poissons fumés retrouvés sur leur étalage selon la langue maitrisée (lingala, kituba ou lari) afin de faciliter la communication et recueillir les informations fiables.

3.6 Enquête sur la consommation du poisson fumé dans les ménages: Nous avons réalisé une enquête transversale de consommation du poisson fumé dans les ménages à Brazzaville, plus précisément dans les arrondissements Bacongo et Ouénzé. Ce qui a permis de connaitre les poissons fumés les plus consommés à Brazzaville. Les informations ont été recueillies à partir d'une fiche d'enquête (annexe 2) portant des questions semi fermées et ouvertes : Les informations ont porté sur :

- $\quad$ Les renseignements généraux ;

- La consommation du poisson fumé ;
- Le mode de consommation ou de préparation des poissons fumés ;

La fréquence de consommation des poissons fumés ;

- L'appréciation du poisson fumé sur le plan organoleptique.

3.7 Type d'étude : Il s'agit d'une étude qui consiste à évaluer la consommation des poissons fumés à Brazzaville et de déterminer les poissons fumés les plus consommés.

3.7.1 Population cible : La population cible est constituée par les femmes et les hommes de 20 ans et plus (ayant la maitrise des recettes familiales) dans les différents ménages et / ou familles à Bacongo et Ouénzé.

\subsubsection{Critères d'inclusion}

- $\quad$ Accepter de participer à l'enquête ;

- Résider dans chacun des arrondissements

Bacongo et Ouénzé au moment de l'enquête ;

- S'occuper habituellement des repas.

3.8 Déroulement de l'enquête : L'enquête dans les ménages s'est déroulée pendant six jours dans les deux arrondissements (six quartiers). Dans chaque arrondissement, une zone d'étude a été délimitée pour la détermination de l'échantillon. Cette détermination s'est faite par tirage aléatoire. La sélection des ménages dans la zone d'étude s'est faite d'une parcelle à une autre de chaque ruelle avec un pas de 2 lorsqu'on longe la ruelle. Ce qui a permis de trouver 120 ménages constituant la taille de notre échantillon. Lors de cette enquête, la personne s'occupant habituellement des repas était interrogée. Une fiche d'information rédigée en français et transcrite en langue nationale, lingala et kituba était présentée à chaque enquêté selon la langue maitrisée. Ce qui permet une bonne compréhension du questionnaire et obtenir des informations fiables. Dans l'arrondissement de Bacongo, les informations ont été généralement recueillies en lari et à ouénzé, elles ont été recueillies en lingala.

3.9 Traitement et analyse des données : Le traitement statistique des données récoltées ainsi que la saisie et la production des tableaux bruts et figures ont été réalisés en utilisant les logiciels EPIINFO. 6.04d fr-2001, Word et Excel 2013. Les variables quantitatives sont exprimées en chiffres et en pourcentage. Les variables qualitatives sont exprimées sous forme de moyenne(x) écart-type (ET). La significativité des différences perçues entre deux pourcentages est vérifiée selon les tests 
classiques de la statistique (test de Khi deux et de student). Le seuil de signification statistique a été 4 RÉSULTATS

4.1 Enquête d'inventaire des poissons fumés vendus dans les marches de Brazzaville (Total, Mampassi et Moungali)

4.1.1 Nombre de vendeurs des poissons fumés inventoriés: Le nombre de vendeurs des poissons fumés recensés est présenté dans le tableau fixé à 5\%.

1. Il ressort de l'analyse des résultats du tableau 1 , que 208 vendeurs correspondant aux 208 étalages de poissons fumés sont inventoriés dont 41 $(19,71 \%)$ au marché Moungali ; $55(26,44 \%)$ au marché de Mampassi et 112 (53,85\%) au marché Total.

Tableau 1 : Répartition du nombre de vendeurs des poissons fumés par marché.

\begin{tabular}{|l|c|c|}
\hline Marchés & Nombre de vendeurs & Pourcentage (\%) \\
\hline Total & 112 & 53,85 \\
Moungali & 41 & 19,71 \\
Mampassi & 55 & 26,44 \\
Total & 208 & 100 \\
\hline
\end{tabular}

4.1.2 Types de poissons fumés vendus sur les marchés: Le tableau 2 présente les types de poissons fumés trouvés et vendus dans les trois marchés enquêtés à Brazzaville. Nous avons noté le nom scientifique et vernaculaire de chacun d'eux.

Tableau 2 : Différents types de poissons fumés généralement vendus sur les marchés enquêtés

\begin{tabular}{|c|c|c|c|c|c|}
\hline \multirow[t]{2}{*}{ origine } & \multirow[t]{2}{*}{$\mathbf{N}^{\circ}$} & \multicolumn{2}{|c|}{ Type de poissons fumés inventoriés } & \multirow[t]{2}{*}{ Effectif } & \multirow{2}{*}{$\begin{array}{c}\text { Pourcentage } \\
(\%)\end{array}$} \\
\hline & & Nom scientifique & nom en Lingala & & \\
\hline \multirow{16}{*}{$\begin{array}{l}\text { Poissons } \\
\text { fumés } \\
\text { d'eau } \\
\text { douce }\end{array}$} & 1 & Clarias spp & Ngolo & 94 & 45,19 \\
\hline & 2 & Protopterus dolloï & Nzombo & 130 & 62,50 \\
\hline & 3 & Distichodus spp & Mboto & 101 & 48,55 \\
\hline & 4 & Parachanna insignis & Moungoussou & 88 & 42,31 \\
\hline & 5 & Labeo spp & Mongandza & 57 & 27,40 \\
\hline & 6 & Chryschthys spp & Machoiron & 7 & 3,36 \\
\hline & 7 & Citharinus spp & Liyanga & 20 & 9,61 \\
\hline & 8 & Heterotis niloticus & Congo ya Sika & 26 & 12,50 \\
\hline & 9 & Papyrocranus congoensis & Peke & 16 & 7,69 \\
\hline & 10 & Hydrocynus spp & Mbenga & 8 & 3,84 \\
\hline & 11 & Anchenoglanis occidentalis & Mboka & 37 & 17,78 \\
\hline & 12 & Mormyrops spp & Mbesse & 33 & 15,86 \\
\hline & 13 & Bariillus & Mombete & 1 & 0,48 \\
\hline & 14 & Tilapia spp & Liboundou & 2 & 0,96 \\
\hline & 15 & Hepsetus microlepis & Mouengue & 3 & 1,44 \\
\hline & 16 & Malapterurus microstoma & Nina & 6 & 2,88 \\
\hline \multirow{7}{*}{$\begin{array}{l}\text { Poissons } \\
\text { fumés de } \\
\text { mer }\end{array}$} & 17 & Cbeilopogon spp & Avion & 20 & 9,61 \\
\hline & 18 & Sardinella spp & Masoundi & 13 & 6,25 \\
\hline & 19 & Scomber japonicus & Maquerelle, Fume & 7 & 3,36 \\
\hline & 20 & - & Bilebelebe & 5 & 2,40 \\
\hline & 21 & Brama & Brama & 1 & 0,48 \\
\hline & 22 & Gadus morbua & Moru Fume & 4 & 1,92 \\
\hline & 23 & Clupéa harengus & Makouala & 4 & 1,92 \\
\hline
\end{tabular}

L'analyse des résultats du tableau 2 montre que, 23 types de poissons fumés ont été inventoriés, dont
16 types de poissons d'eau douce et 7 types de poissons de mer. Les poissons les plus vendus dans 
ces marchés sont : Protopterus dolloï « Nzombo », Distichodus spp «Mboto», Clarias spp " Ngolo », Parachanna insignis « Moungoussou » avec un pourcentage respectif de 62,$50 ; 48,55 ; 45,19$ et 42,31. Viennent ensuite: les Labeo spp «Mongandza», Heterotis niloticus «Congo ya Sika», Anchenoglanis occidentalis «Mboka», Mormyrops spp «Mbesse» avec un pourcentage respectif de vente de
27,$40 ; 12,50 ; 17,78$ et 15,86 . Le pourcentage de vente des autres poissons étant très faible.

4.2 Enquête de consommation des poissons fumes.

4.2.1 Age et niveau d'instruction des enquêtés : Les informations recueillies sur l'âge et le niveau d'instruction des individus enquêtés sont indiquées dans le tableau 3.

Tableau 3: Répartition des enquêtés selon l'âge et le niveau d'instruction

\begin{tabular}{|c|c|c|c|c|c|}
\hline paramètres & variables & Effectif & $(\%)$ & IC à 95\% & Valeur du $\chi^{2} \mathrm{p}$ \\
\hline \multirow[t]{2}{*}{ Age des enquêtés } & $\begin{array}{l}\text { 20-25ans } \\
26-30 a n s \\
31-35 a n s \\
36-40 a n s \\
41-45 a n s \\
46-50 a n s \\
51-55 a n s \\
\text { 56-60ans } \\
60 \text { ans et plus }\end{array}$ & $\begin{array}{c}12 \\
27 \\
14 \\
15 \\
14 \\
14 \\
7 \\
4 \\
13 \\
\end{array}$ & $\begin{array}{c}10,0 \\
22,5 \\
11,7 \\
12,5 \\
11,7 \\
11,7 \\
5,8 \\
3,3 \\
10,80\end{array}$ & $\begin{array}{c}5,3 \%-16,8 \% \\
15,4 \%-31,0 \% \\
6,5 \%-18,8 \% \\
7,2 \%-19,8 \% \\
6,5 \%-18,8 \% \\
6,5 \%-18,8 \% \\
2,4 \%-11,6 \% \\
0,9 \%-8,3 \% \\
5,9 \%-17,8 \%\end{array}$ & $\begin{array}{c}\chi^{2}=19,053 \\
P<0,001\end{array}$ \\
\hline & \multicolumn{4}{|c|}{ Moyenne \pm ET : $39,29 \pm 11,93$ ans } & \\
\hline $\begin{array}{l}\text { niveau } \\
\text { d'instruction }\end{array}$ & $\begin{array}{l}\text { Primaire } \\
\text { Collège } \\
\text { Lycée } \\
\text { Supérieure } \\
\text { Jamais scolarisé }\end{array}$ & $\begin{array}{l}9 \\
36 \\
34 \\
31 \\
10\end{array}$ & $\begin{array}{c}7,5 \\
30,0 \\
28,3 \\
25,8 \\
8,4\end{array}$ & $\begin{array}{c}3,5 \%-13,8 \% \\
22,0 \%-39,0 \% \\
20,5 \%-37,3 \% \\
18,3 \%-34,6 \% \\
4,1 \%-14,8 \%\end{array}$ & $\begin{array}{c}\chi^{2}=29,737 \\
p<0,001\end{array}$ \\
\hline
\end{tabular}

Il ressort du tableau 3, les observations suivantes : Concernant l'âge des enquêtés, il varie entre 20 à 60ans. La tranche d'âge la plus représentée est celle de 26 à 30 ans $(22,5 \%)$; suivie de celle dont l'âge est compris entre 36-40ans (12,5\%). La tranche d'âge la moins représentée est celle dont l'âge est compris entre 56-60ans (3,3\%). L'âge moyen des enquêtés est de 39,29 $\pm 11,93$ ans. La différence est très significative car $\mathrm{p}<0,001$. S'agissant du niveau d'instruction, la majorité des personnes enquêtées est scolarisée, puisque seulement $8,4 \%$ d'enquêtés ne sont jamais scolarisés. Le niveau d'instruction des enquêtés le plus représenté est celui du collège avec un pourcentage de 30,0 ; suivi du niveau lycée avec un pourcentage de 28,3; puis du niveau supérieur avec un pourcentage de 25,8 et enfin le niveau primaire avec un pourcentage de 7,5. La différence est très significative car la probabilité $\mathrm{p}<0,001$.

4.2.2 Genre des personnes enquêtées: Il ressort du tableau 4 que sur 120 personnes enquêtées, il y a plus de personnes du genre féminin $(80 \%)$ que de personnes du genre masculin $(20 \%)$. La différence est très significative car la probabilité $\mathrm{p}<0,001$.

Tableau 4: présente le genre des personnes enquêtées.

\begin{tabular}{|c|c|c|c|c|}
\hline Genre & Effectif & $(\%)$ & IC à $95 \%$ & Valeur de $\chi^{2}, p$ \\
\hline Masculin & 24 & 20,0 & \multirow{3}{*}{$\begin{array}{l}13,3 \%-28,3 \% \\
71,7 \%-86,7 \%\end{array}$} & \multirow{3}{*}{$\begin{array}{c}\chi^{2}=49,089 \\
\mathrm{p}<0,001\end{array}$} \\
\hline Féminin & 96 & 80,0 & & \\
\hline Total & 120 & 100,0 & & \\
\hline
\end{tabular}


4.2.3 Statut sociodémographique et socioéconomique : Le tableau 5 illustre les résultats sur le statut sociodémographique et socio-économique des enquêtés.

Tableau 5: Répartition des enquêtés selon statut sociodémographique et socio-économique

\begin{tabular}{|c|c|c|c|c|c|}
\hline Paramètres & Variables & Effectif & $(\%)$ & IC à 95\% & Valeur de $\chi^{2}, P$ \\
\hline \multirow{5}{*}{$\begin{array}{c}\text { Situation } \\
\text { matrimoniale }\end{array}$} & Célibataire & 49 & 40,8 & $32,0 \%-50,2 \%$ & \multirow{5}{*}{$\begin{array}{c}\chi^{2}=19,444 \\
p<0,001\end{array}$} \\
\hline & Divorcé & 3 & 2,5 & $0,5 \%-7,1 \%$ & \\
\hline & Union libre & 21 & 17,5 & $11,2 \%-25,5 \%$ & \\
\hline & Marié & 36 & 30,0 & $22,0 \%-39,0 \%$ & \\
\hline & Veuf (ve) & 11 & 9,2 & $4,7 \%-15,8 \%$ & \\
\hline \multirow{11}{*}{ Taille du ménage } & 1pers & 5 & 4,1 & $1,4 \%-9,5 \%$ & \multirow{11}{*}{$\begin{array}{c}\chi^{2}=22,322 \\
p<0,001\end{array}$} \\
\hline & 2 pers & 14 & 11,7 & $6,5 \%-18,8 \%$ & \\
\hline & 3 pers & 18 & 15,0 & $9,1 \%-22,7 \%$ & \\
\hline & 4pers & 24 & 20,0 & $13,3 \%-28,3 \%$ & \\
\hline & 5 pers & 17 & 14,2 & $8,5 \%-21,7 \%$ & \\
\hline & 6pers & 18 & 15,0 & $9,1 \%-22,7 \%$ & \\
\hline & 7 pers & 5 & 4,2 & $1,4 \%-9,5 \%$ & \\
\hline & 8pers & 6 & 5,0 & $1,9 \%-10,6 \%$ & \\
\hline & 9 pers & 4 & 3,3 & $0,9 \%-8,3 \%$ & \\
\hline & Sup à 9 pers & 9 & 7,5 & $3,5 \%-13,8 \%$ & \\
\hline & \multicolumn{4}{|c|}{ Moyenne \pm ET : 4,875 $\pm 2,392$} & \\
\hline \multirow{4}{*}{$\begin{array}{c}\text { Nombre des } \\
\text { participants à la } \\
\text { popote }\end{array}$} & 1pers & 108 & 90,0 & $83,2 \%-94,7 \%$ & \multirow{4}{*}{$\begin{array}{c}\chi^{2}=20,021 \\
\mathrm{p}<0,001\end{array}$} \\
\hline & 2 pers & 6 & 5,0 & $1,9 \%-10,6 \%$ & \\
\hline & 3 pers & 1 & 0,8 & $0,0 \%-4,6 \%$ & \\
\hline & Sup à 3pers & 5 & 4,2 & $1,4 \%-9,5 \%$ & \\
\hline \multirow{4}{*}{ Profession } & Fonctionnaire & 19 & 15,8 & $9,8 \%-23,6 \%$ & \multirow{4}{*}{$\begin{array}{c}\chi^{2}=31,185 \\
p<0,001\end{array}$} \\
\hline & Secteur privé & 19 & 15,8 & $9,8 \%-23,6 \%$ & \\
\hline & Informel & 59 & 49,2 & $39,9 \%-58,4 \%$ & \\
\hline & Commerçant & 23 & 19,2 & $12,6 \%-27,4 \%$ & \\
\hline
\end{tabular}

L'analyse des résultats du tableau 5 montre les faits suivants. Concernant la situation matrimoniale, les résultats montrent qu'il y a 49 ménages (40,8\%) enquêtés dont le chef de ménage est célibataire, suivi de 36 ménages $(30 \%)$ dont le chef de ménage est marié et 21 ménages $(17,5 \%)$ dont le chef vit en union libre. Les classes les moins représentées sont celles des veufs $(9,2 \%)$ et des divorcés $(2,5 \%)$. La différence est très significative car la probabilité $\mathrm{p}<0,001$. Pour la taille des ménages, les résultats montrent qu'elle est variable. Les ménages ayant 4 personnes sont plus représentés avec un pourcentage de 20, suivi des ménages ayant 3 et 6 personnes avec un pourcentage de 15. Enfin, ceux ayant 5 personnes avec un pourcentage de 14,2. Les ménages ayant 9 personnes sont les moins représentés avec un pourcentage de 3,3. La différence est très significative car la probabilité $\mathrm{p}<0,001$. Concernant la popote, les résultats montrent que dans la plupart des ménages enquêtés, une seule personne ne participe à la popote, soit $90 \%$. La différence est très significative car la probabilité $\mathrm{p}<0,001$. Enfin pour la profession, il ressort que la population enquêtée a un niveau socio-économique moyen car sur l'ensemble d'individus enquêtés, 19,2\% sont commerçants, 15,8 $\%$ sont fonctionnaires, $15,8 \%$ travaillant dans un secteur privé et 49,2\% exercent une activité informelle. La différence est très significative car la probabilité $\mathrm{p}<0,001$.

4.2.4 Consommation et choix du poisson fumé : Les résultats sur la consommation et le choix du poisson fumé par les personnes soumises à l'enquête sont présentés dans le tableau 6. 
Tableau 6 : Répartition des enquêtés selon la consommation et le choix du poisson fumé

\begin{tabular}{|l|c|c|c|c|c|}
\hline Paramètres & Variables & Effectif & $\mathbf{( \% )}$ & I C à 95\% & Valeur de $\boldsymbol{\chi}^{2}, \mathbf{p}$ \\
\hline $\begin{array}{l}\text { Consommation du } \\
\text { poisson fumé }\end{array}$ & Oui & 105 & 87,5 & $80,2 \%-92,8 \%$ & \\
& Non & 15 & 12,5 & $7,2 \%-19,8 \%$ & $\begin{array}{c}\chi^{2}=37,108 \\
\mathrm{p}<0,001\end{array}$ \\
\hline $\begin{array}{l}\text { Choix du poisson } \\
\text { fume }\end{array}$ & Oui & 65 & 61,9 & $50,9 \%-70,3 \%$ & $\begin{array}{c}\chi^{2}=29,066 \\
\mathrm{p}<0,001\end{array}$ \\
\hline
\end{tabular}

Il ressort de l'analyse des résultats du tableau 6, les faits suivants. Concernant la consommation du poisson fumé, les résultats montrent que sur 120 individus enquêtés, 105 individus (soit 87,5\%) consomment le poisson fumé et $15(12,5 \%)$ n'en consomment pas. La différence est très significative car la probabilité $\mathrm{p}<0,001$. S'agissant du choix du poisson fumé, les résultats montrent que parmi les ménages consommant le poisson fumé, $65(61,5 \%)$ choisissent le poisson fumé consommé et 40 $(38,1 \%)$ n'en choisissent pas. La différence est très significative car la probabilité $\mathrm{p}<0,001$.

4.2.5 Critères de choix du poisson fumé : Les critères de choix du poisson fumé par les consommateurs sont présentés à la figure 2 .

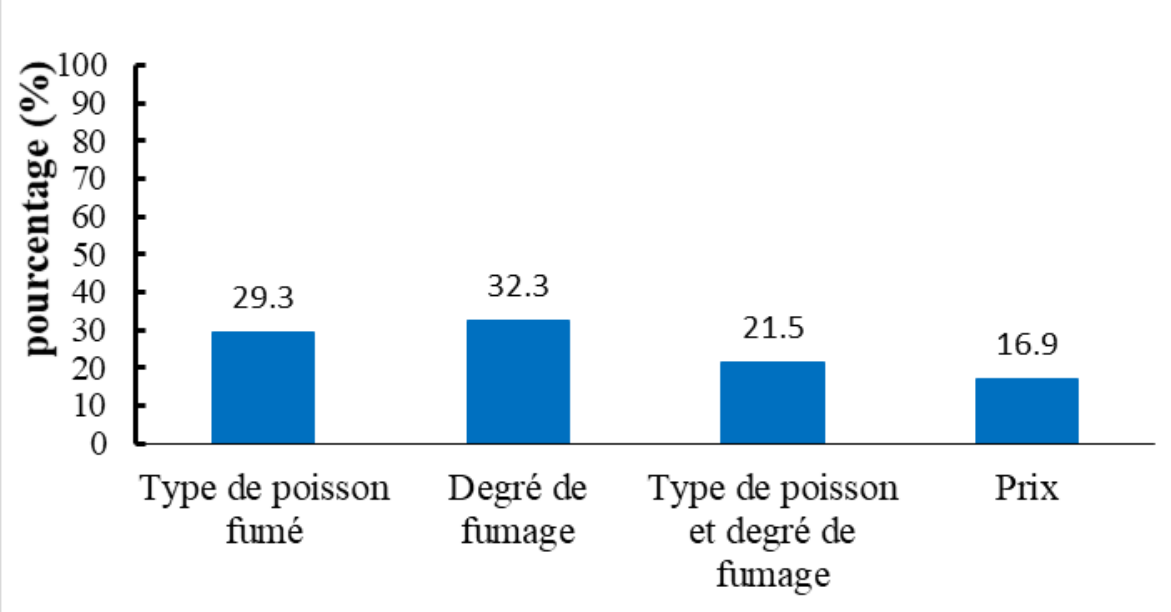

Figure 2 : Répartition des consommateurs en fonction des critères de choix du poisson fumé

La figure 2 montre que 32,3\% des enquêtés choisissent le poisson fumé par rapport au degré de fumage, $29,3 \%$ le choisissent selon le type de poisson, $21,5 \%$ en choisissent selon le degré de fumage et le type de poisson. Enfin, $16,9 \%$ des ménages choisissent le poisson fumé par rapport à son prix de vente dans le marché. La différence est très significative car la probabilité $\mathrm{p}<0,001$.

4.2.6 Types de poissons fumés souvent consommés par les enquêtés: Les types de poissons fumés souvent consommés par les enquêtés sont présentés à la figure 3 . 


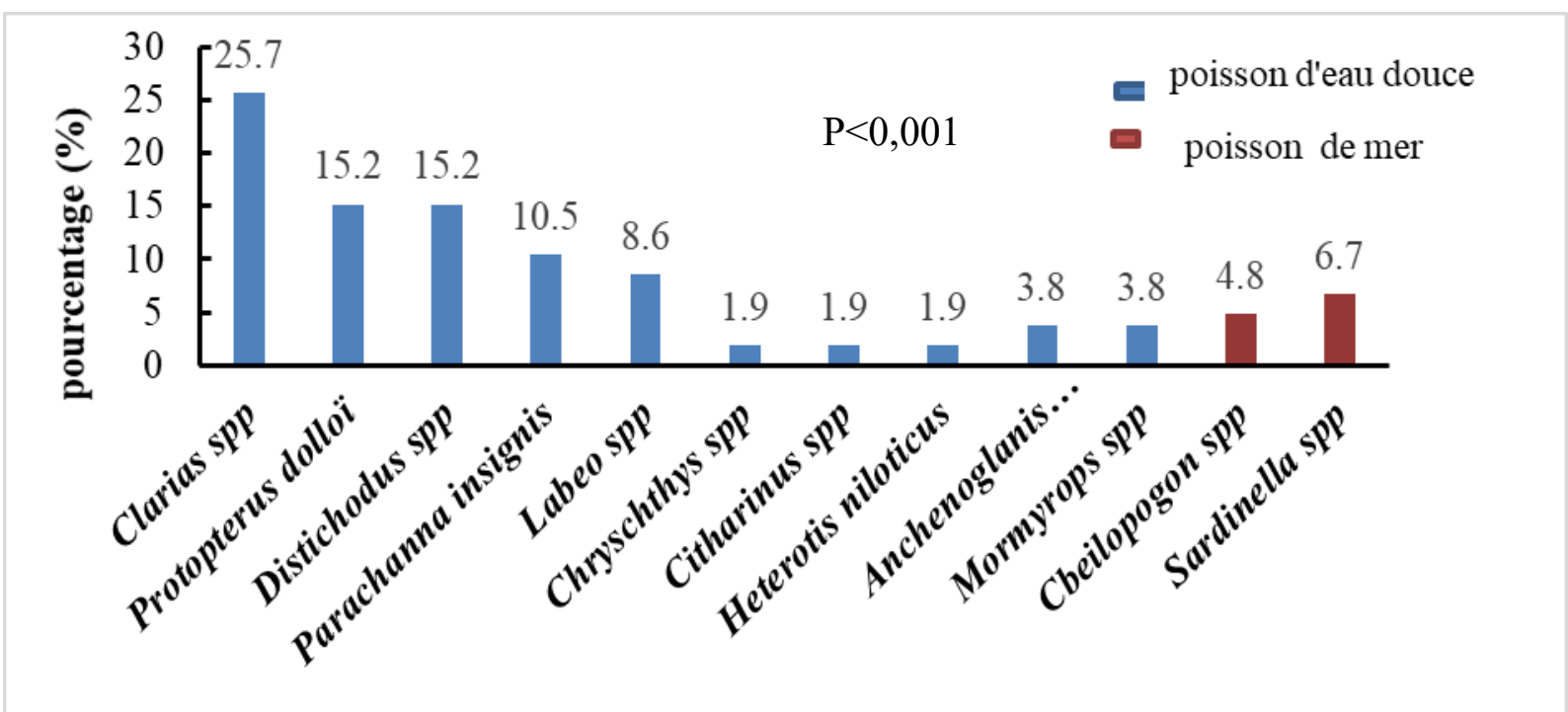

Figure 3 : Répartition des enquêtés en fonction des types de poissons fumés souvent consommés

Les résultats de la figure 3 montrent que $88,5 \%$ des enquêtés consomment souvent le poisson d'eau douce fumé et $11,5 \%$ des enquêtés consomment souvent le poisson de mer fumé. Ainsi les trois poissons fumés les plus consommés par les ménages enquêtés sont Clarias spp «Ngolo» (25,7\%), suivi de Protopterus dolloï « Nzombo»
$(15,2 \%)$ et de Distichodus spp «Mboto » (15,2\%). La différence est très significative car la probabilité $\mathrm{p}<0,001$.

4.2.7

Types de poissons fumés préférés par les consommateurs : La figure 4 présente les résultats sur les types de poissons fumés préférés dans les ménages.

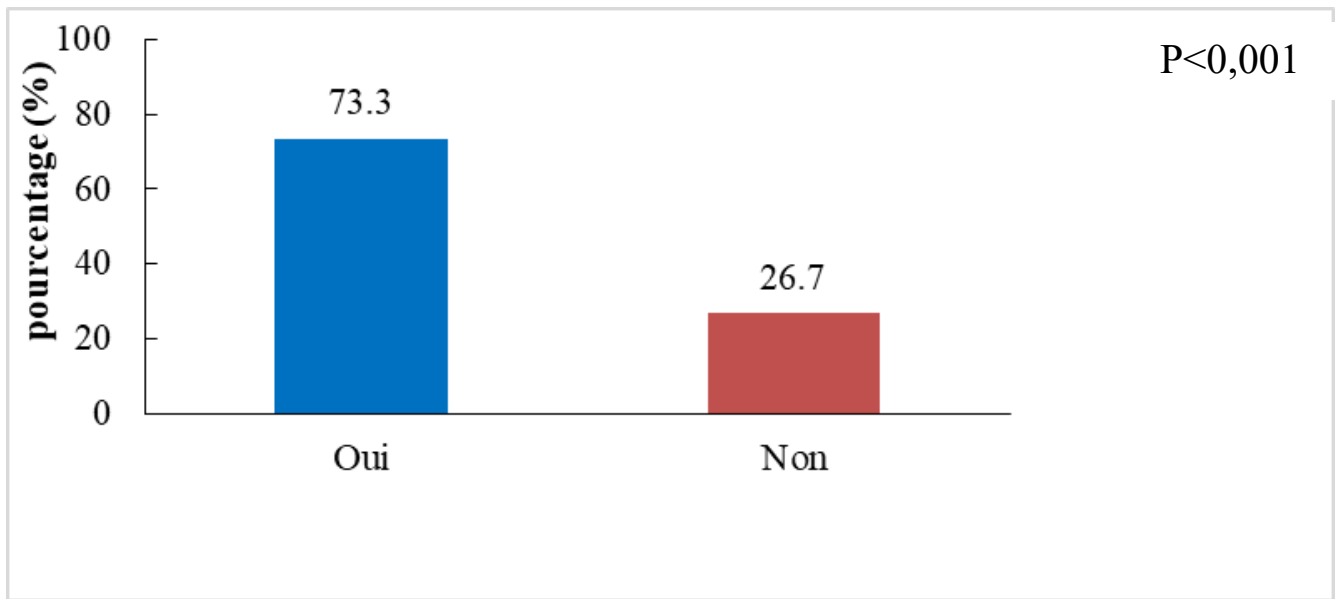

Figure 4: Répartition des consommateurs en fonction des types de poissons fumés préférés

Il ressort de ces résultats que, sur l'ensemble des ménages enquêtés, $73,3 \%$ consomment souvent le type du poisson fumé préféré et $26,7 \%$ n'en consomment pas. La différence est très significative car la probabilité $\mathrm{p}<0,001$.
4.2.8

Raisons

de non consommation du poisson fumé préféré : $\mathrm{La}$ figure 5 illustre les raisons pour lesquelles certains enquêtés ne consomment pas souvent le type de poisson fumé préféré. 


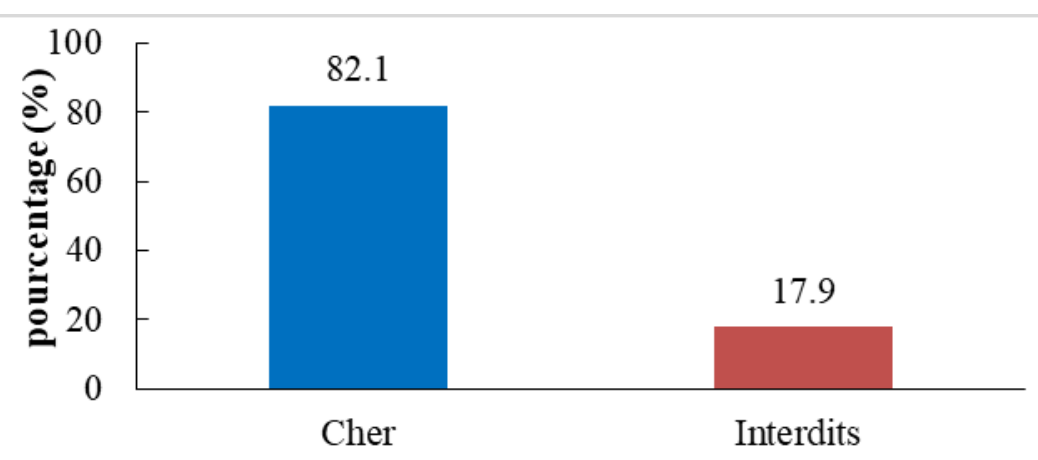

$\mathrm{P}<0,001$

Figure 5 : Répartition des enquêtés en fonction des raisons de non consommation du poisson fumé préféré

Les résultats de la figure 5 montrent que, certains ménages $(82,1 \%)$ ne consomment pas souvent le type du poisson fumé préféré parce que ces poissons sont chers au marché. Leur revenu financier ne permet pas l'achat du type de poisson fumé préféré. D’autres par contre $(17,9 \%)$, ne consomment pas souvent le poisson fumé préféré à cause des interdits et des habitudes alimentaires. La différence est très significative car la probabilité $\mathrm{p}<0,001$.

4.2.9 Consommation du poisson fumé par tous les membres de la famille : Les informations recueillies concernant l'ampleur de la consommation par tous les membres de la famille sont données dans le tableau 7.

Tableau 7: Ampleur de la consommation du poisson fumé dans le ménage

\begin{tabular}{|l|c|c|c|c|}
\hline $\begin{array}{l}\text { Consommation du poisson fume } \\
\text { par tous les membres de la } \\
\text { famille }\end{array}$ & Effectif & (\%) & IC à 95\% & Valeur de $\chi^{2}, \mathbf{p}$ \\
\hline Oui & 95 & 90,5 & $83,2 \%-95,3 \%$ & \\
Non & 10 & 9,5 & $4,7 \%-16,8 \%$ & $\chi^{2}=38,050$ \\
TOTAL & 105 & 100,0 & & $\mathrm{p}<0,001$ \\
\hline
\end{tabular}

Les résultats du tableau 7 montrent que sur l'ensemble des ménages consommant le poisson fumé, celui-ci est consommé à 90,5\% par tous les membres du ménage contre $9,5 \%$ qui ne le consomme pas. La différence est très significative, avec la probabilité $\mathrm{p}<0,001$.
4.2.10 Nombre de personnes ne consommant pas le poisson fumé dans les ménages: Les résultats sur le nombre de personnes ne consommant pas le poisson fumé dans les ménages consommateurs sont présentés dans le tableau 8.

Tableau 8: Répartition du nombre de personnes ne consommant pas le poisson fumé

\begin{tabular}{|l|c|c|c|c|}
\hline $\begin{array}{l}\text { Nombre de personnes ne } \\
\text { consommant pas le poisson } \\
\text { fume dans le ménage }\end{array}$ & Effectif & $\mathbf{( \% )}$ & IC à 95\% & Valeur de $\chi^{2}, \mathbf{p}$ \\
\hline 1 pers & 4 & 40,0 & $12,2 \%-73,8 \%$ & \\
2 pers & 3 & 30,0 & $6,7 \%-65,2 \%$ & $\chi^{2}=5,284$ \\
$>3$ pers & 3 & 30,0 & $6,7 \%-65,2 \%$ & $\mathrm{p}<0,001$ \\
TOTAL & 10 & 100,0 & & \\
\hline
\end{tabular}

Les résultats du tableau 8 montrent que, parmi les ménages où le poisson fumé n'est pas consommé par tous les membres, $40 \%$ de ces ménages ont une personne ne consommant pas le poisson fumé, suivi de $30 \%$ des ménages ayant deux personnes et de $30 \%$ de ménages ayant au plus trois personnes n'en consommant pas. La différence est très significative avec une probabilité $\mathrm{p}<0,001$. 
4.2.11 Formes de consommations du poisson fumé : Les formes de consommation du poisson fumé sont présentées à la figure 6 .

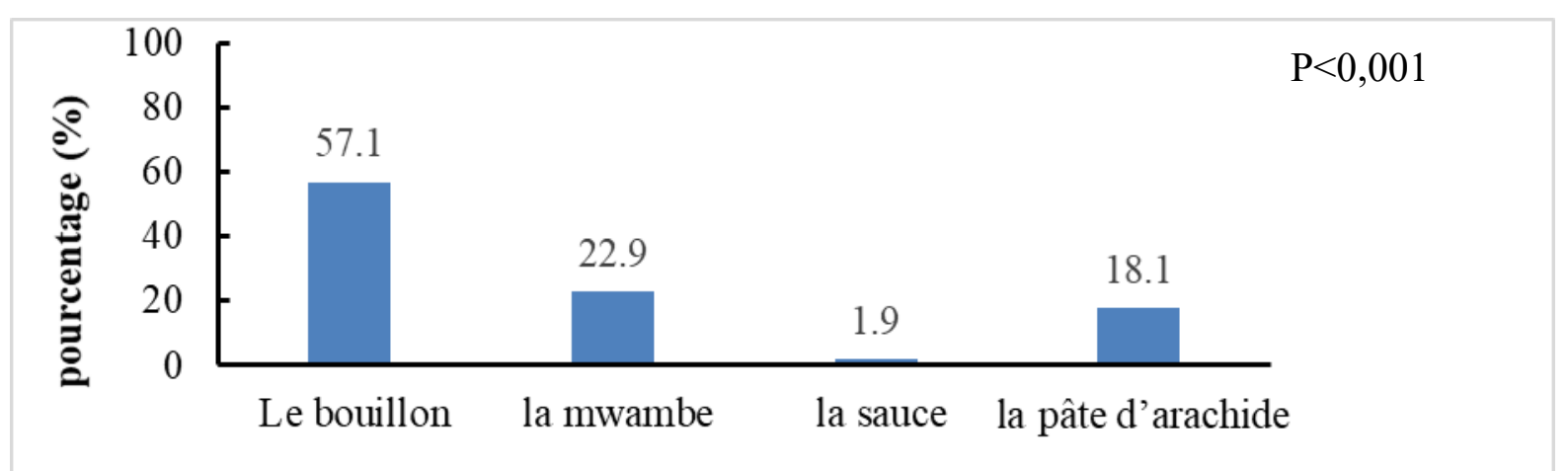

Mwambe $=$ jus des noix de palme

Figure 6: Répartition des enquêtés selon les formes de consommation du poisson fumé

La figure 6 montre que $57,1 \%$ des ménages enquêtés consomment souvent le poisson fumé sous forme de bouillon et 22,9\% le consomment souvent avec de la mwambe et 18,1 \% le consomment souvent avec la pâte d'arachide. La consommation sous la forme sauce est moins pratiquée. La différence est très significative car la probabilité $\mathrm{p}<0,001$.

4.2.12 Formes préférées de consommation du poisson fumé: Les formes préférées de consommation du poisson fumé par les enquêtés sont présentées à la figure 7 .

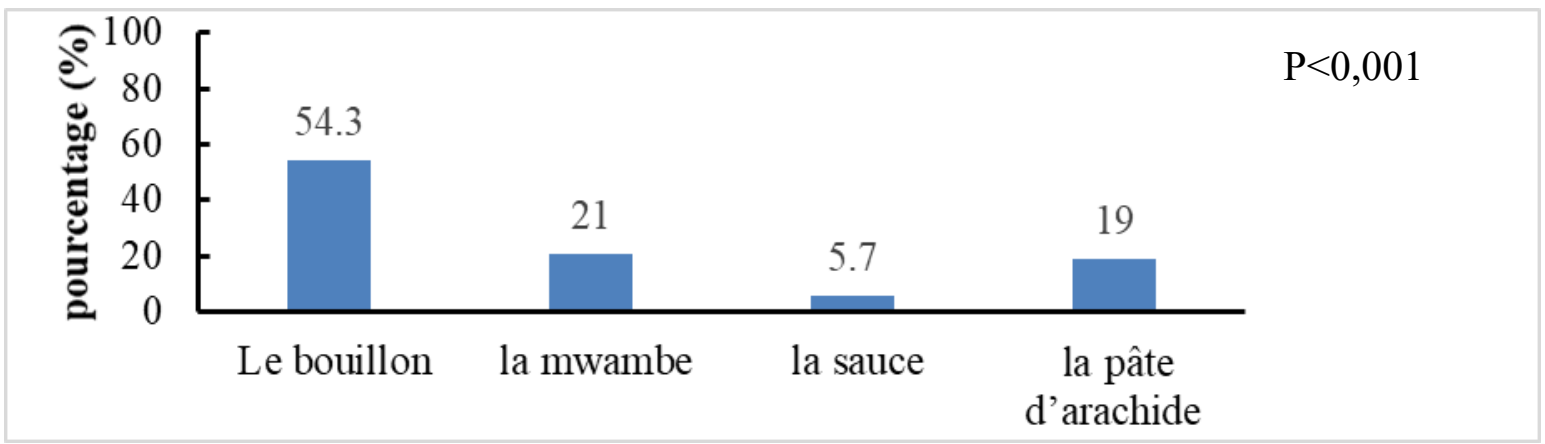

Mwambe $=$ jus des noix de palme

Figure 7 : Répartition des enquêtés selon les formes préférées de consommation du poisson fumé

La figure 7 montre que 54,3\% des ménages enquêtés préfèrent consommer le poisson fumé sous forme de bouillon, $21 \%$ le préfèrent avec de la mwambe et $19 \%$ avec la pâte d'arachide. La forme est moins préférée. La différence est très significative car la probabilité $\mathrm{p}<0,001$.
4.2.13 Aliments accompagnant souvent la consommation du poisson fumé : Les aliments accompagnant la consommation du poisson fumé sont présentés à la figure 8 . 


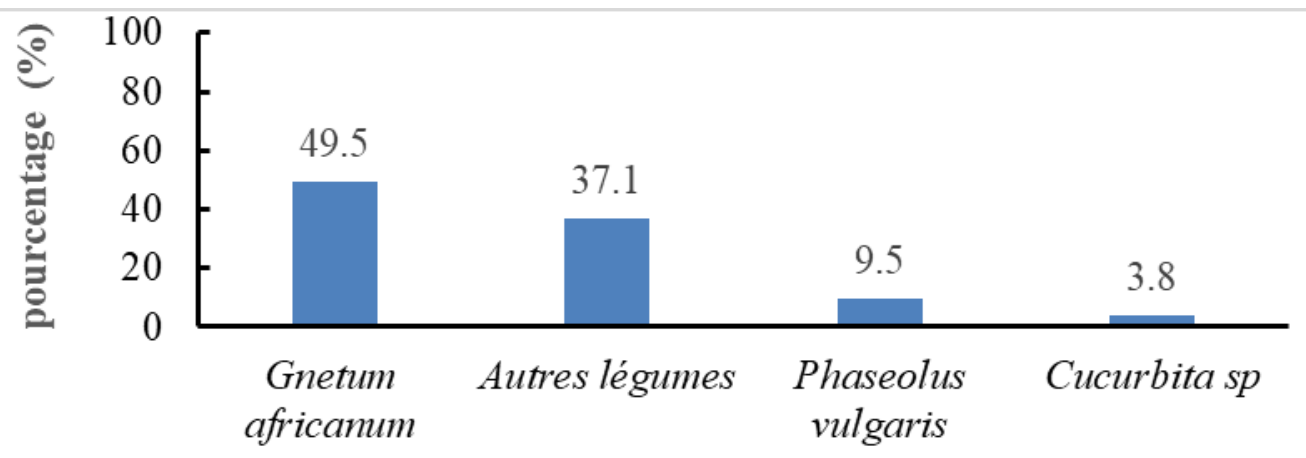

Figure 8 : Répartition des aliments accompagnant la consommation du poisson fumé selon les enquêtés

Les résultats montrent que tous les individus enquêtés consommant du poisson fumé, en l'accompagnant avec d'autres aliments. Il ressort de l'analyse de la figure 8 que, sur 105 individus consommant le poisson fumé, 49,5\% d'individus l'accompagnent souvent avec Gnetum africanum «koko », 37,1 \% d'individus accompagnent le poisson fumé avec d'autres légumes (endive, épinard, aubergines, Cucurbita lagenaria, oseille...),
9,5\% d'individus l'accompagnent avec Phaseolus vulgaris (haricot) et 3,8\% avec Cucurbita sp (courge). La différence est très significative, car la probabilité $\mathrm{p}<0,001$.

4.2.14 Aliments préférés accompagnant la consommation du poisson fumé : Les résultats concernant les aliments préférés accompagnant la consommation du poisson fumé sont consignés à la figure 9 .

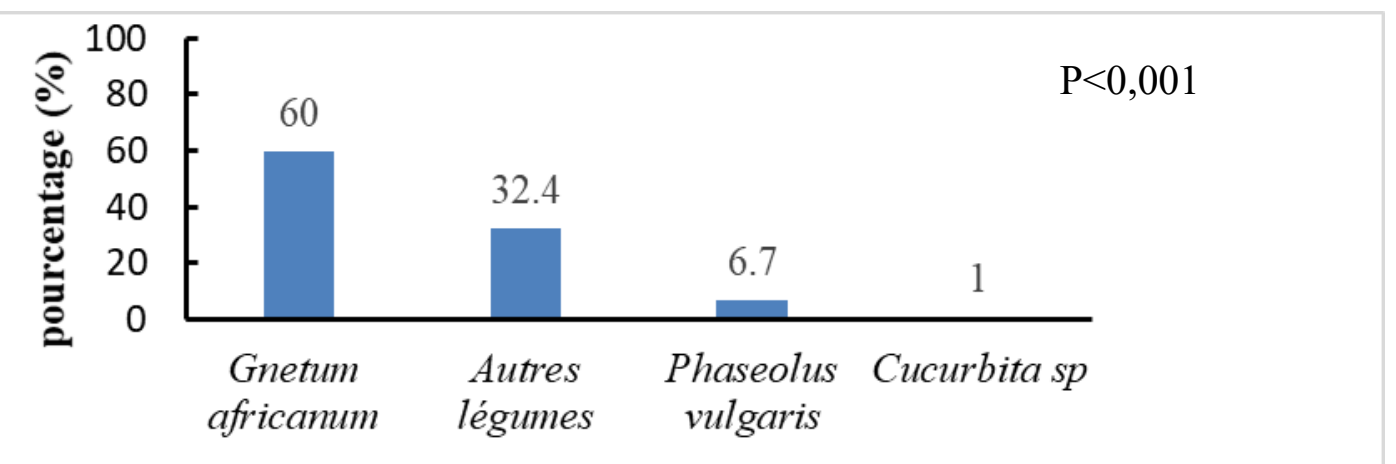

Figure 9 : Répartition des aliments préférés accompagnant la consommation du poisson fumé en fonction des enquêtés

Les résultats montrent que, $60 \%$ des individus enquêtés préfèrent consommer le poisson fumé avec Gnetum africanum «koko », 32,4 \% préfèrent le consommer avec d'autres légumes (endive, épinard, aubergines, Cucurbita lagenaria, oseille...), 6,7 \% préfèrent le consommer avec Phaseolus vulgaris (haricot) et $1 \%$ préfèrent le consommer avec Cucurbita sp (courge). La différence est très significative, car la probabilité $\mathrm{p}<0,001$.

4.2.15 Fréquence de consommation du poisson fumé : La figure 10 présente la fréquence hebdomadaire de consommation du poisson fumé. 


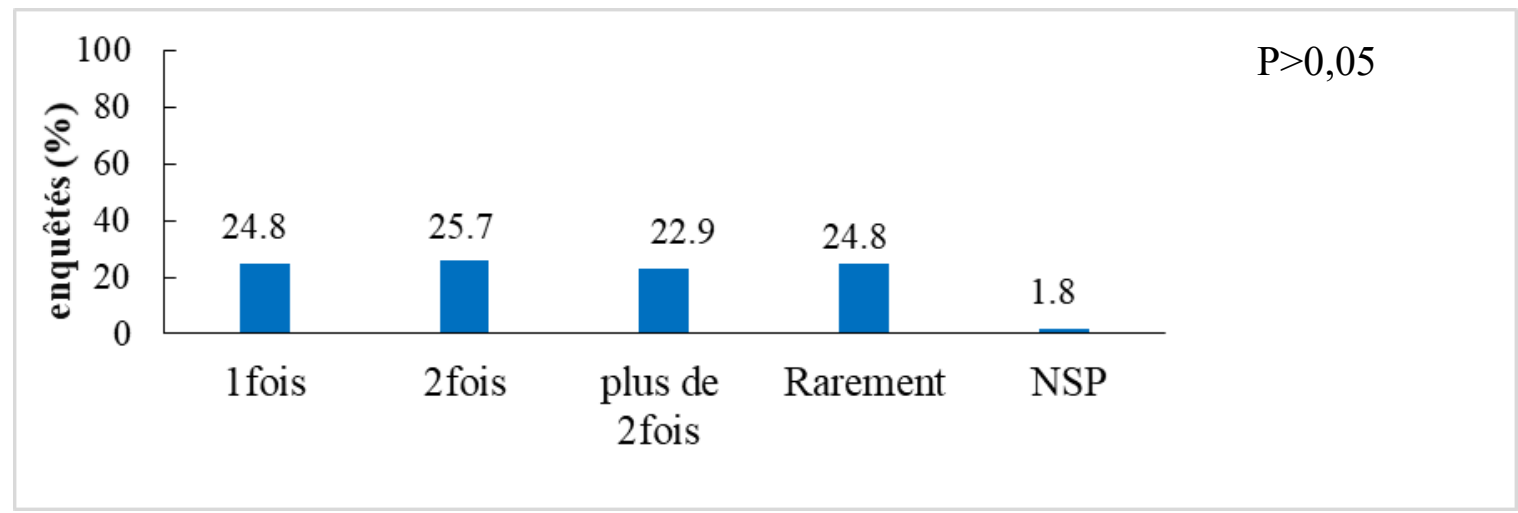

Figure 10 : Répartition de la fréquence de consommation du poisson fumé

Les résultats montrent que, la fréquence de consommation du poisson fumé varie selon les ménages. En effet, $25,7 \%$ des ménages consomment du poisson fumé 2 fois dans la semaine; $24,8 \%$ en consomment 1 fois dans la semaine; $24,8 \%$ consomment le poisson fumé rarement ; 22,9\% consomment le poisson fumé plus de 2 fois dans la semaine et 1,8\% ne se rappellent pas. La différence est non significative, car la probabilité $\mathrm{p}>0,05$.

4.2.16 Dernière consommation $\mathrm{du}$ poisson fumé : Les résultats concernant la dernière fois où le poisson fumé est consommé sont présentés à la figure 11.

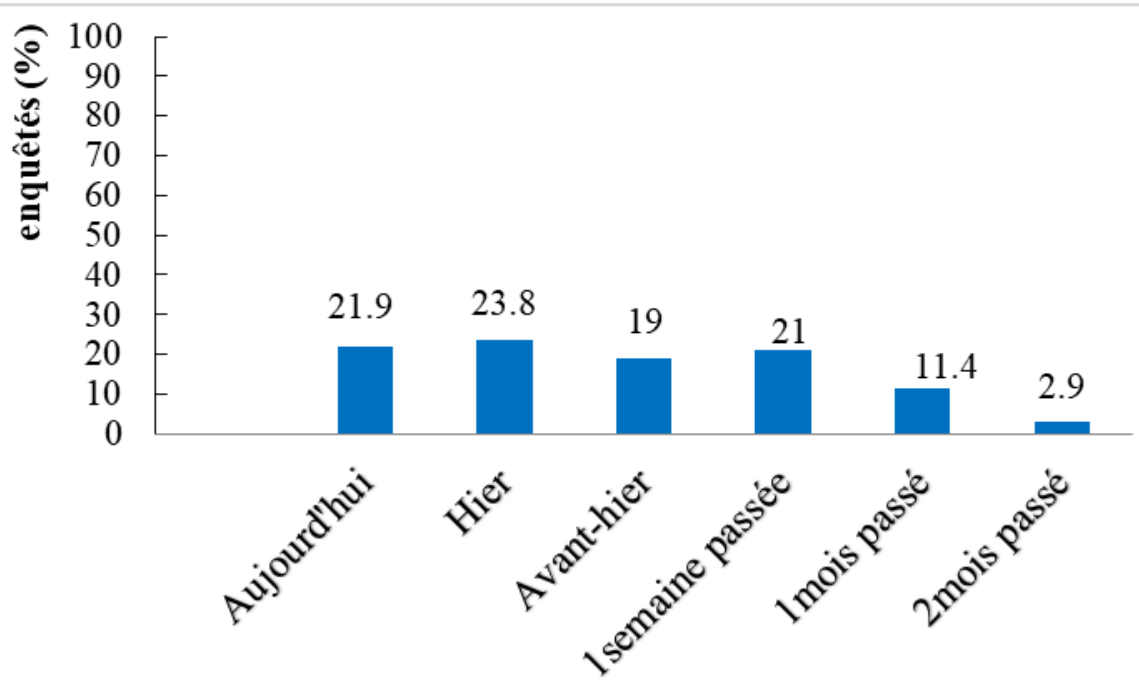

Figure 11 : Répartition de la dernière consommation du poisson fumé en fonction des enquêtés

Les résultats de la figure 11 montrent que, parmi les consommateurs enquêtés, $23,8 \%$ ont consommé le poisson fumé $24 \mathrm{~h}$ avant l'enquête (la veille de l'enquête), 21,9\% l'ont consommé le jour de l'enquête; 21,0\% l'ont consommé une semaine avant l'enquête $19 \%$ l'ont consommé $48 \mathrm{~h}$ avant l'enquête, $11,4 \%$ l'ont consommé un mois avant l'enquête et 2,9\% l'ont consommé deux mois avant l'enquête. La différence est non significative, car la probabilité $\mathrm{p}>0,05$.

4.2.17 Caractéristiques organoleptiques du poisson fumé : Les qualités organoleptiques du poisson fumé sont présentées dans le tableau 9. 
Tableau 9: Caractéristiques organoleptiques du poisson fumé

\begin{tabular}{|c|c|c|c|c|}
\hline Qualités sensorielles & Effectif & $(\%)$ & IC à 95\% & Valeur de $\chi^{2}, p$ \\
\hline \multicolumn{5}{|l|}{ GOUT } \\
\hline$\overline{B o n}$ & 103 & 98,1 & $93,3 \%-99,8 \%$ & \multirow{3}{*}{$\begin{array}{c}\chi^{2}=76,027 \\
p<0,001\end{array}$} \\
\hline Mauvais & 2 & 1,9 & $0,2 \%-6,7 \%$ & \\
\hline TOTAL & 105 & 100,0 & & \\
\hline \multicolumn{5}{|l|}{ COULEUR } \\
\hline Bon & 103 & 98,1 & $93,3 \%-99,8 \%$ & \multirow{3}{*}{$\begin{array}{c}\chi^{2}=76,027 \\
p<0,001\end{array}$} \\
\hline Mauvais & 2 & 1,9 & $0,2 \%-6,7 \%$ & \\
\hline TOTAL & 105 & 100,0 & & \\
\hline \multicolumn{5}{|l|}{ ODEUR } \\
\hline Bon & 96 & 91,4 & $84,4 \%-96,0 \%$ & \multirow{3}{*}{$\begin{array}{c}\chi^{2}=39,552 \\
p<0,001\end{array}$} \\
\hline Mauvais & 9 & 8,6 & $4,0 \%-15,6 \%$ & \\
\hline TOTAL & 105 & 100,0 & & \\
\hline
\end{tabular}

Les résultats du tableau 9 montrent que la plupart des ménages enquêtés apprécient le poisson fumé comme un bon aliment avec un bon goût $(98,1 \%)$,

\section{DISCUSSION}

Cette étude avait pour objectif général d'évaluer la consommation et la qualité nutritionnelle des poissons fumés à Brazzaville, Il s'agissait spécifiquement d'inventorier les poissons fumés vendus à Brazzaville et de Connaitre les poissons fumés consommés à Brazzaville. Concernant l'inventaire des poissons fumés vendus à Brazzaville, notre étude montre qu'il existe une diversité des poissons fumés vendus dans les trois marchés enquêtés. En effet, 23 types des poissons fumés sont inventoriés lors de cette étude. Soulignons que, certaines espèces n'ont pas été trouvées sur les marchés pendant la période de notre enquête; c'est le cas de "Mopongo; Ngulu masa». Ceci pourrait s'expliquer soit par des contraintes liées au fumage (teneur élevée en lipide); soit, par leur écologie (cycle de reproduction). Ces résultats sont en accord avec ceux obtenus par Mujinga et al. (2009) sur l'inventaire des espèces de poissons disponibles sur les marchés urbains à Lubumbashi et Mujinga (2009) sur les catégories des produits de la pêche commercialisés aux marchés de Lubumbashi et leur valeur nutritionnelle. En effet, ces auteurs ont constaté une diversité des poissons vendus; mais, certaines espèces étaient introuvables. De même Gsegner et al (2009) réalisant une étude sur le marché de Niomo au Niger, avaient constaté une très grande variété d'espèces commercialisée sous les formes, fumée, frais, séché et frite. Mais, certaines espèces étaient absentes notamment sous une bonne couleur $(98,1 \%)$ et une bonne odeur $(91,4 \%)$. La différence est très significative car $\mathrm{p}<0,001$.

la forme fumée ; c'est le cas des poissons Chryschthys (C. auratus et C. nigrodigitatus), Polypterus senegalus. Notre étude montre que sur les 23 types de poissons fumés inventoriés, les poissons d'eau douce fumés sont plus vendus (16 types) que les poissons de mer fumés (7 types). Ces résultats rejoignent ceux de Le Gall et Petit jean (1975) sur la commercialisation des poissons à Brazzaville qui évoquent que l'offre en poissons de mer fumés y est très faible. S'agissant de la consommation du poisson fumé à Brazzaville, nos résultats montrent qu'il est très consommé dans les ménages enquêtés $(87,5 \%)$. Nos résultats rejoignent l'affirmation de Opoye-Itoua (1989) qui évoque que le poisson est la principale source des protéines animales au Congo. Notre étude montre que les poissons fumés sont consommés avec une importance variable dont, les poissons d'eau douce fumés sont souvent plus consommés $(88,5 \%$ ) que les poissons de mer fumés $(11,5 \%)$. Par contre, ils diffèrent de ceux obtenus par Grosse (2009) à la suite d'une étude réalisée au Cameroun qui révèle que, la majorité des poissons les plus consommés au centre du Cameroun est d'origine marine (78\%) et non continentale $(4,2 \%)$. Constatons, que les ménages enquêtés préfèrent plus les poissons d'eau douce fumés $(92,5 \%)$ que les poissons de mer fumés $(7,5 \%)$. Dans le même sens, ce constat avait été fait par Le Gall et Petit Jean (1975), qui ont montré que $46 \%$ des Brazzavillois préfèrent le poisson d'eau douce fumé contre $4 \%$ pour le 
poisson de mer fumé. Il ressort de nos résultats que le poisson fumé est consommé par 49,2\% des enquêtés ayant un niveau socio-économique bas contre $50,8 \%$ ayant un niveau socio-économique moyen. Disons que la consommation du poisson fumé, ne dépend pas des revenus de la population. Bien que ce dernier pourrait influencer la consommation en termes de qualité (type de poisson) ou de quantité. Ce constat rejoint l'affirmation de la FAO (2009) selon laquelle: « Le poisson fumé constitue une source importante de protéines animales accessible aux ménages à faibles revenus, surtout dans les pays en voie de développement où le prix de la viande demeure hors de la portée du consommateur moyen ».

\section{CONCLUSION}

Cette étude a révélé qu'il existe une diversité des poissons fumés vendus et consommés à Brazzaville. La consommation du poisson fumé à Brazzaville dépend de sa disponibilité dans le marché. Le poisson fumé en particulier le poisson d'eau douce fumé occupe une place de choix dans l'alimentation

\section{REFERENCES BIBLIOGRAPHIQUES}

Diènèba Keita Ouattara. Contribution à l'étude de la qualité des poissons transformés (fumés, séchés) .Ouattara D.M.Université de Bamako.Thèse de Pharmacie.2005.139p.

FAO. Document technique sur les pêches et l'aquaculture. $2009.65 \mathrm{p}$

FAO. L'état de l'insécurité alimentaire dans le monde. Organisation des Nations Unies pour l'alimentation et l'agriculture. Rome 2003. $36 \mathrm{p}$.

FAO. Profil des pêches et de l'aquaculture par pays ; vue générale du secteur des pêches nationales. FAO/FIR/CP/BEN. 2006. $42 \mathrm{p}$.

FAO. Systèmes d'information et de cartographie sur l'insécurité alimentaire et la vulnérabilité (SICIAV). FAO. Rome. 2000.40p.

Fray R. Livre d'or du centenaire de Brazzaville.Brazzaville.1980. 354p.

Gbaguidi P et Fiogbe I. Rapport national sur la pêche au Bénin.ACP-EU Training. 1998. $8 \mathrm{p}$.

Gram L. Spoilage of three Senegalese fish species stored in ice and at ambient temperature. Paper presented at SEAFOOD in Halifax, Canada. 1987.198p.
Concernant la fréquence de consommation des poissons fumés, nos résultats montrent, qu'elle varie selon les ménages à savoir: 25,7\% des ménages consomment du poisson fumé deux fois dans la semaine ; $24,8 \%$ en consomment une fois dans la semaine et $22,9 \%$ consomment le poisson fumé plus de deux fois dans la semaine. Ces résultats se rapprochent de ceux trouvés par Mujinga et al. (2009) à Lubumbashi, lesquels a montré que la fréquence de consommation du poisson en général à Lubumbashi varie selon les ménages d'une fois à sept fois par semaine. Nos résultats montrent que les poissons fumés les plus vendus (Clarias spp, Protopterus dollö et Distichodus spp) sont les plus consommés et les plus préférés.

des habitants de Brazzaville. Il s'agit particulièrement du poisson fumé Clarias spp « Ngolo », Protopterus dollö « Nzombo » et Distichodus spp«mboto», avec une fréquence de consommation souvent de deux fois par semaine

Grosse O. La place du poisson dans la consommation alimentaire des villageois des régions Centre et Ouest du Cameroun. APDRA-F. France. 2009. 25p

Gsegner C, Bentz B et Forgo Y. Etude préalable à un test de la pisciculture dans la zone Office du Niger. A P D R A - F Association Pisciculture et Développement Rural en Afrique - France. 2009. 41p

Le Gall et Petit Jean. Commercialisation du poisson à Brazzaville : résultat d'un sondage.Pointe-Noire: ORSTOM. 1975.31p.

Liston J. Bacterial spoilage of seafood. Quality Assurance in the Fish Industry. Proceedings of an International conférence, Copenhagen, Denmark, Elsevier. 1992. 93105 p.

Mujinga W. Catégories des produits de la pêche commercialisés aux marchés de Lubumbashi et leur valeur nutritionnelle. Les pêches et le VIH/SIDA en Afrique: investir dans les solutions durables. République Démocratique du Congo. 2009. $23 \mathrm{p}$.

Mujinga W., lwambaj, Mutala S. et Hüsken S. M. C. Inventaire des espèces de poisson 
disponibles sur les marchés urbains de Lubumbashi. Les pêches et le VIH/SIDA en Afrique: investir dans les solutions durables. République Démocratique du Congo. 2009. 30p.

Opoye - Itoua. Production et consommation du poisson fumé (Mokalu) au Congo: aspects techniques, hygiéniques et socio économiques. Thèse, Médecine vétérinaire.Université cheikh Anta diop. Dakar. 1989. 170p.

WorldFish center. Le poisson et la sécurité alimentaire en Afrique. WorldFish Center, Penang (Malaisie). 2005. 11p

ANNEXE1 : Fiche d'inventaire des poissons fumés vendus dans les marchés de Brazzaville

UNIVERSITE MARIEN NGOUABI
FACULTE DES SCIENCES ET TECHNIQUES
DEPARTEMENT DES MASTERS
PARCOURS : BIOLOGIE
SOUS PARCOURS : PHYSIOLOGIE ANIMALE
ETUTRITION

FICHE D'INVENTAIRE

ENQUETE EXHAUSTIVE DANS LES MARCHES DE BRAZZAVILLE

But : faire l'inventaire des poissons fumés vendus dans les marchés de Brazzaville

Nom du Marché :

Date :

\begin{tabular}{|l|l|}
\hline $\mathbf{n}^{\text {o d'étalage }}$ & Type de poisson vendu \\
\hline Etalage 1 & \\
\hline Etalage2 & \\
\hline Etalage3 & \\
\hline Etalage4 & \\
\hline Etalage 5 & \\
\hline Etalage 6 & \\
\hline & \\
Etalage7 & \\
\hline & \\
\hline
\end{tabular}


ANNEXE 2 : Fiche d'enquête de consommation du poisson fumé dans les ménages

UNIVERSITE MARIEN NGOUABI
FACULTE DES SCIENCES ET TECHNIQUES
DEPARTEMENT DES MASTERS
PARCOURS : BIOLOGIE
SOUS PARCOURS : PHYSIOLOGIE ANIMALE
ET NTTRITION

Titre du thème : Caractérisation de la consommation alimentaire des poissons fumés à Brazzaville

Questionnaire de consommation

Numéro du dossier...........................................................

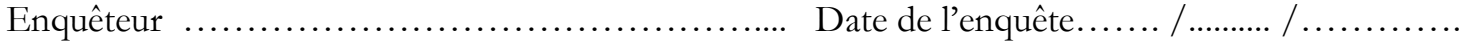

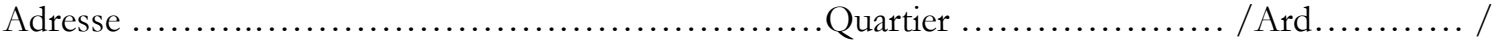

\begin{tabular}{|c|c|c|}
\hline No & Caractères & code \\
\hline I & Renseignement généraux & \\
\hline 1 & 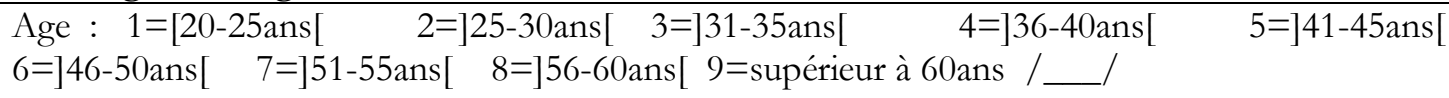 & \\
\hline 2 & $\begin{array}{l}\text { Niveau d'instruction : } 1=\text { primaire } ; 2=\text { collège ; } 3=\text { lycée } ; \quad 4=\text { supérieur } \quad 5= \\
\text { jamais. }\end{array}$ & \\
\hline 3 & Genre $: 1=$ Masculin /__/ 2=féminin /__/ & \\
\hline 4 & 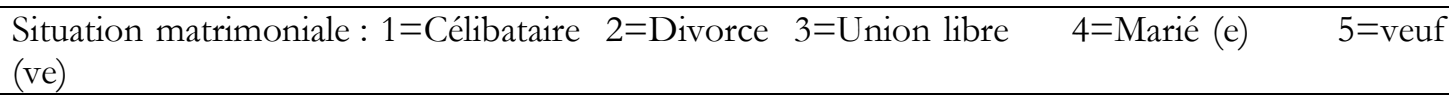 & \\
\hline 5 & Profession : $\quad 1=$ fonctionnaire $; 2=$ secteur privé $; 3=$ informel $; 4=$ commerçant & \\
\hline 6 & $\begin{array}{l}\text { Nombre de personne dans le ménage : } 1=1 \text { pers } 2=2 \text { pers } 3=3 \text { pres } 4=4 \text { pres } 5=5 \text { pers } \\
6=6 \text { pres } 7=7 \text { pers } 8=8 \text { pers } 9=9 \text { pres } 10=10 \text { pers }\end{array}$ & \\
\hline 7 & Combien participe à la popote? & \\
\hline II & Consommation du poisson fumé & \\
\hline 8 & Consommez-vous le poisson fumé ? $1=$ oui $2=$ non $/$ & \\
\hline 9 & Choisissez-vous le poisson que vous consommez ? $1=$ oui $2=$ non $/$ & \\
\hline 10 & $\begin{array}{l}\text { Si oui, quels sont les critères de choix : } 1=\text { type du poisson fumé ? } \\
2=\text { degré de fumage } 3=\text { type et le degré de fumage } 4=\text { prix }\end{array}$ & \\
\hline 11 & Quel type de poisson fumé consommez-vous souvent? & \\
\hline 12 & Quel type de poisson fumé préférez-vous consommé? & \\
\hline 13 & Consommez-vous souvent le type préféré ? $1=$ oui $2=$ non & \\
\hline 14 & Si non donné la raison.... & \\
\hline 15 & Tous les membres du ménage consomment-ils le poisson fumé ? $1=$ oui ; $2=$ non & \\
\hline
\end{tabular}




\begin{tabular}{|c|c|}
\hline 16 & Si non combien en consomme (nt)? \\
\hline III & Mode de consommation ou de préparation \\
\hline 17 & $\begin{array}{l}\text { sous quelle forme consommez-vous le poisson fumé ? } 1=\text { bouillon } 2=\text { à la mwambe } 3=\text { à la } \\
\text { sauce } 4=\text { à la pâte d'arachide } 5=\text { non préparé ; } 6=\text { toute les formes }\end{array}$ \\
\hline 18 & $\begin{array}{l}\text { Sous quelle forme préférez-vous consommer le poisson fumé ? } 1=\text { bouillon } 2=\text { à la mwambe } \\
3=\text { à la sauce } 4=\text { à la pâte d'arachide } 5=\text { non préparé }\end{array}$ \\
\hline 19 & $\begin{array}{l}\text { Accompagnez-vous la consommation du poisson fumé avec d'autres aliments ? } \\
\begin{array}{cl}1=\text { oui } \quad 2=\text { non }\end{array}\end{array}$ \\
\hline 20 & $\begin{array}{l}\text { si oui, avec quel aliment accompagnez-vous le poisson fumé ? } 1=\text { autres légumes } 2=\text { Gnetum } \\
\text { africaum } 3=\text { haricot } 4=\text { courge }\end{array}$ \\
\hline 21 & $\begin{array}{l}\text { quel est votre aliment d'accompagnement préféré? } 1=\text { autres légumes } 2=\text { Gnetum africaum } \\
3=\text { haricot } 4=\text { courge }\end{array}$ \\
\hline IV & Fréquence de consommation \\
\hline 22 & $\begin{array}{l}\text { 13-Combien de fois consommez-vous le poisson fumé dans la semaine } \\
1=\text { une fois } \quad 2=\text { deux fois } \quad 3=\text { plus de } 2 \text { fois } \quad 4=\text { rarement } 5=\text { NSP }\end{array}$ \\
\hline 23 & $\begin{array}{l}\text { Quand avez-vous consommé pour la dernière fois le poisson fumé ? } 1=\text { semaine passée ; } \\
2=1 \text { mois passé } ; 3=2 \text { mois passé } ; \\
4=\text { hier } ; 5=\text { avant-hier } ; 6=\text { aujourd'hui }\end{array}$ \\
\hline 24 & la fréquence de consommation reste constante toute l'année ? $1=$ oui ; $2=$ non ; $3=$ ignoré \\
\hline $\mathbf{V}$ & Appréciation du poisson fumé sur le plan organoleptique \\
\hline 25 & $\begin{array}{l}\text { Comment trouvez-vous le goût du poisson fumé ? } 1=\text { bon } 2=\text { mauvais } \\
3=\text { autre (précisez) }\end{array}$ \\
\hline 26 & $\begin{array}{l}\text { Comment trouvez-vous la couleur du poisson fumé ? 1=bon 2=mauvais } \\
3=\text { autre (précisez) }\end{array}$ \\
\hline 27 & $\begin{array}{l}\text { Comment trouvez-vous l'odeur du poisson fumé ? } 1=\text { bon } 2=\text { mauvais } \\
3=\text { autre (précisez) }\end{array}$ \\
\hline
\end{tabular}

